\title{
Descripción de una especie nueva de alacrán con importancia médica del género Centruroides (Scorpiones: Buthidae) del estado de Colima, México
}

\section{Description of a new species of scorpion of medical importance of the genus Centruroides (Scorpiones: Buthidae) from the state of Colima, Mexico}

\author{
Javier Ponce-Saavedra ${ }^{1}$ y Oscar F. Francke ${ }^{2 *}$ \\ ${ }^{1}$ Laboratorio de Entomología “Biol. Sócrates Cisneros Paz," Facultad de Biología, Universidad Michoacana de San Nicolás de Hidalgo, Edificio \\ B-4, $2^{\circ}$ piso, Ciudad Universitaria, 58060 Morelia, Michoacán, México. \\ ${ }^{2}$ Departamento de Zoología, Instituto de Biología, Universidad Nacional Autónoma de México, Apartado postal 70-153, 04510 México, D.F., México. \\ *Correspondencia: offb@ibiologia.unam.mx
}

\begin{abstract}
Resumen. Se describe Centruroides hirsutipalpus sp. nov. de la región de Minatitlán, Colima. Se compara con las especies morfológica y geográficamente más cercanas, C. elegans Thorell y C. tecomanus Hoffmann y con otras especies "rayadas" de Centruroides del centro-occidente de México. Es ésta una especie con importancia médica que no se había recolectado previamente.
\end{abstract}

Palabras clave: Buthidae, Centruroides, especie nueva, sistemática, Colima, México.

\begin{abstract}
Centruroides hirsutipalpus sp. nov. from the region of Minatitlán, Colima, Mexico is described. The new species is compared with C. elegans Thorell and C. tecomanus Hoffmann, which are morphologically and geographically closely related. Comparisons with other species of "striped" Centruroides from central and western Mexico are included. This medically important species had not been collected previously.
\end{abstract}

Key words: Buthidae, Centruroides, new species, systematics, Colima, Mexico.

\section{Introducción}

Los alacranes son un grupo de animales con importancia médica y biológica. Se conocen cerca de 1450 especies descritas en el mundo (Fet et al., 2000; Rein, 2008). Actualmente se ha incrementado su estudio no sólo toxicológico y sistemático, sino también el ecológico y biológico (Polis, 1990; Polis y Sissom, 1990; Brown, 2001; Brown et al., 2002; Ponce, 2003; Yamashita, 2004; Ponce et al., 2003; Contreras-Garduño et al., 2006). México es el país más rico en el mundo, aproximadamente con 216 especies descritas (15\% del total mundial) que corresponden a 23 géneros pertenecientes a las 7 familias reconocidas para América del Norte (Buthidae, Chactidae, Diplocentridae, Euscorpiidae, Iuridae, Superstitioniidae y Vaejovidae), con 17 subespecies y al menos 52 taxones que esperaban ser descritos para 2001 (Beutelspacher, 2000; Fet et al., 2000, González-Santillán, 2001; GonzálezSantillán s.f.), quedando aún áreas geográficas importantes sin estudiar.

De la familia Buthidae, hasta 1998 se habían registrado

Recibido: 27 noviembre 2008; aceptado: 03 febrero 2009 en el mundo 73 géneros, 529 especies y 165 subespecies (Fet et al., 2000), número que se ha incrementado con la descripción de 8 géneros y 119 especies (Rein, 2008; Ponce et al., 2009; Santibáñez-López y Ponce, 2009). En México, el género Centruroides Marx 1890 es el más común y diverso. En general se consideraba como el único representante de la familia Buthidae; sin embargo, De Armas y Martín-Frías (1998) describieron Tityopsis aliciae con un ejemplar bastante deteriorado procedente de la zona de Tehuantepec, Oaxaca. La presencia de esta especie en el país ha sido corroborada recientemente (Vidal y Francke, 2009).

En la actualidad, el género Centruroides contiene 65 especies y unas 10 subespecies del Nuevo Mundo. Para México se han registrado 29 especies, 5 con taxones subespecíficos (De Armas et al., 2003; Ponce y Moreno, 2005; Francke y Sissom, inédito). Los alacranes como grupo se consideran animales de amplia distribución; sin embargo, en el nivel específico es relativamente común encontrar distribuciones restringidas; incluso, a escalas locales, las especies muestran patrones de distribución espacial claramente influenciados por las preferencias de microhábitat (Polis, 1990). 
El género Centruroides se distribuye desde el sur de los Estados Unidos hasta América Central y el norte de América del Sur (Fet et al., 2000). México tiene especies con distribuciones muy amplias, como $C$. infamatus (Koch) y C. limpidus (Karsch) en el centro del país, $C$. gracilis (Latreille) en el sureste, C. exilicauda (Wood) en la península de Baja California y C. vittatus (Say) en el noreste (Beutelspacher, 2000; González-Santillán, 2001). De acuerdo con González-Santillán (2001), en el género se reconocen 4 grupos de especies: a) grupo "rayados", especies con 8 hileras o series de granulaciones en el borde interno de los dedos de los pedipalpos y con 2 bandas longitudinales oscuras flanqueando una banda amarilla mesal que le dan la apariencia rayada; $b$ ) grupo "bertholdii", especies con 8 hileras o series de granulaciones en el borde interno de los dedos de los pedipalpos y dorsalmente sin "rayas"; c) grupo "gracilis", especies con 9 hileras de granulaciones en el borde interno de los pedipalpos, y d) grupo "thorelli", especies de tamaño pequeño, con pigmentación en forma de manchas, sin llegar a constituir bandas, y con hábitos arborícolas. En Colima se tiene registro de 8 especies de alacranes (una dudosa), de las cuales 2 pertenecen a Centruroides: C. tecomanus y $C$. infamatus (Hoffmann, 1931, 1932, Beutelspacher, 2000, Fet et al., 2000; Ponce y Moreno, 2005; Ponce et al., 2009); aunque no hay acuerdo sobre la presencia de la subespecie C. infamatus ornatus, la cual sólo la registra Beutelspacher (2000) (Cuadro1).

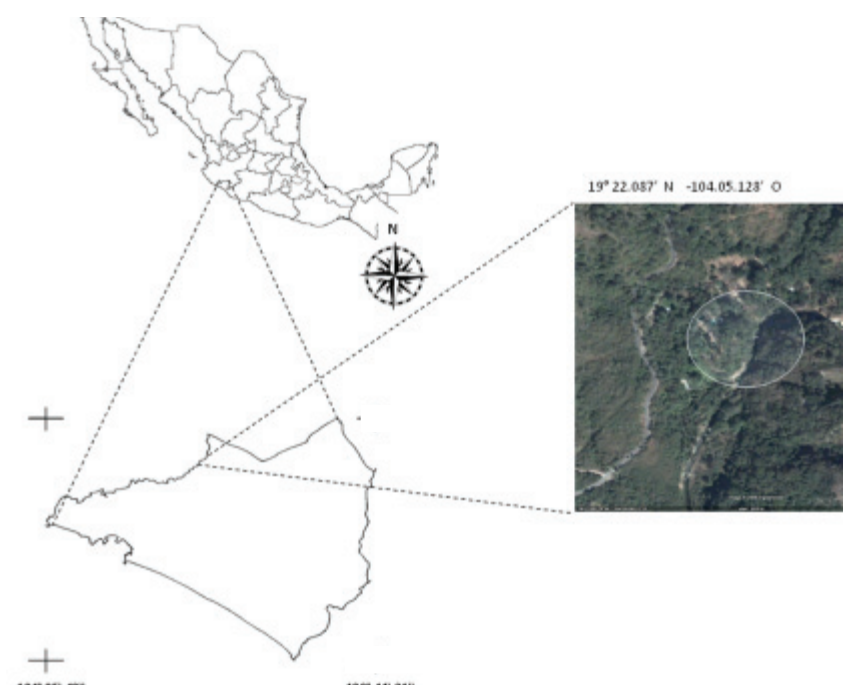

Figura 1. Ubicación de la localidad tipo. Modificado de Google Earth ${ }^{\circledR}$.

\section{Materiales y métodos}

Se revisaron 8 ejemplares adultos (3 hembras y 5 machos) provenientes de la localidad Balneario El Salto en el municipio de Minatitlán, Colima, ubicada a los $19^{\circ}$ $22.087^{\prime} \mathrm{N}$ y $104^{\circ} 05.128^{\prime} \mathrm{O}$, a una altitud de $650 \mathrm{~m}$, en el occidente de México (Fig. 1). Los alacranes fueron recolectados de noche utilizando lámparas de detección de

Cuadro 1. Especies de alacranes registradas para el estado de Colima

\begin{tabular}{|c|c|c|c|}
\hline Familia & Especie & Subespecie & Fuente \\
\hline \multirow[t]{4}{*}{ Buthidae } & Centruroides elegans (Thorell) & C. elegans elegans (Thorell) & Francke y Sissom, enviado \\
\hline & \multirow[t]{2}{*}{ Centruroides infamatus (C. L. Koch) } & $\begin{array}{l}\text { C. infamatus infamatus (C.L. } \\
\text { Koch) }\end{array}$ & $\begin{array}{l}\text { Hoffmann, 1932; Beutelspacher, } \\
\text { 2000; Fet et al., 2000; Ponce y } \\
\text { Moreno, 2005. }\end{array}$ \\
\hline & & $\begin{array}{l}\text { C. infamatus ornatus } \\
\text { Hoffmann }\end{array}$ & Beutelspacher, 2000 (dudoso) \\
\hline & Centruroides tecomanus Hoffmann & & $\begin{array}{l}\text { Hoffmann, 1932; Beutelspacher, } \\
\text { 2000; Fet et al., 2000; Ponce y } \\
\text { Moreno, 2005; Ponce et al., } 2009\end{array}$ \\
\hline \multirow[t]{6}{*}{ Vaejovidae } & \multirow[t]{2}{*}{ Vaejovis intrepidus Thorell } & V. intrepidus atrox Hoffmann & $\begin{array}{l}\text { Hoffmann, 1931; Díaz-Nájera 1975; } \\
\text { Fet et al., 2000; Beutelspacher } 2000 .\end{array}$ \\
\hline & & $\begin{array}{l}\text { V. intrepidus intrepidus } \\
\text { Thorell }\end{array}$ & $\begin{array}{l}\text { Hoffmann 1931; Diáz-Nájera 1975; } \\
\text { Fet et al. } 2000\end{array}$ \\
\hline & Vaejovis janssi Williams & & Fet et al., 2000; Beutelspacher 2000. \\
\hline & Vaejovis monticola Sissom & & $\begin{array}{l}\text { Sissom, 1989; Fet et al., 2000; } \\
\text { Beutelspacher } 2000 .\end{array}$ \\
\hline & Vaejovis nitidulus & & Beutelspacher, 2000 (dudoso) \\
\hline & Vaejovis sp. aff. kuarapu Francke y Ponce & & Francke, datos no publicados. \\
\hline
\end{tabular}


luz negra, en 2 fechas de colecta: el 26 de mayo de 2006 y el 29 de agosto de 2007. Los ejemplares se sacrificaron por choque térmico y se conservaron en alcohol etílico al $75 \%$ para su traslado al laboratorio.

Se seleccionó un macho adulto como holotipo, los restantes fueron designados paratipos. Todos los ejemplares se revisaron bajo microscopio estereoscópico y se les hicieron 29 mediciones y el conteo pectinal, para obtener la variación morfológica y morfométrica de la especie. Para la nomenclatura y mediciones en la descripción se siguió a Stahnke (1970), excepto en las quillas o carenas metasomales, para las cuales se siguió a Francke (1977) y la propuesta de Vachon (1974, 1975), para la terminología tricobotrial. Las observaciones se hicieron en un microscopio estereoscópico Stemi DV4 y las mediciones con el mismo aparato, auxiliados por un ocular micrométrico de $0.1 \mathrm{~mm}$ de precisión.

\section{Descripción}

\section{Centruroides hirsutipalpus sp.nov.}

\section{Holotipo macho}

Patrón de coloración. Color base amarillo, dorsalmente más oscuro que en el vientre. Patrón típico de los alacranes "rayados", con manchas sobre las placas dorsales las cuales forman 2 bandas oscuras a los lados de una banda mesal clara, las cuales recorren longitudinalmente el mesosoma. Patas completamente claras en la base y sólo ligeramente manchadas dorsolateralmente en tibia y basitarso. Parte distal de manos y dedos, así como la vesícula, de color ligeramente más oscuro que el resto del cuerpo.

Prosoma. Caparazón con 4 líneas longitudinales bien definidas; las externas inician en la parte posterior de los ojos laterales, sin llegar al borde del caparazón y convergen hacia el borde posterior en donde se observa una ligera curvatura hacia el borde externo y se fusionan con una mancha transversal que da inicio a las bandas longitudinales del mesosoma; las medias inician en el borde anterior y son más intensas en el área ocular y pre-ocular, donde se observa un ligero engrosamiento con pigmento intenso y posteriormente disminuye en intensidad y grosor formando líneas divergentes que no alcanzan el borde posterior. Área de ojos laterales intensamente pigmentada. Zona entre ojos laterales y surco medio con pigmentación difusa (que al microscopio hacen poco definidas las líneas). Borde anterior no recto, ligeramente crenulado y se observa levemente recurvado. Superficie con granulación escasa e irregular, mayormente concentrada en la mitad anterior y hacia el área ocular. Bordes laterales ligeramente crenulados, sobre todo en tercio anterior (Fig. 2a).
Mesosoma. Con 2 bandas longitudinales anchas, bien definidas separadas por una banda de color claro, ligeramente más ancha. Bandas formadas por mancha delgada de color intenso en el preterguito y otra más gruesa de forma trapezoidal en el borde posterior del posterguito, unidas por pigmentación más clara y difusa. Bordes laterales con pigmento bien definido en toda la longitud de las placas I y II, pero a partir de la placa III, disminuye la cantidad de pigmento hasta que en las placas VI y VII ya el borde es completamente claro. Banda mesal de color claro, con quilla granular apreciable en posterguitos III-VI, disminuyendo en longitud, quedando solo en la parte posterior de los posterguitos V y VI, en donde los gránulos están mejor definidos que en los terguitos anteriores. Terguito VII bien pigmentado solo en preterguito; en posterguito el pigmento se reduce a las quillas medias laterales, dando la apariencia de 2 líneas delgadas (Fig. 2b); ventralmente 8 sedas bien desarrolladas (Fig.2c). Los peines tienen conteo de 23-23 y la base presenta una escotadura media bien definida, claramente distinta a la de las hembras en las que borde es casi recto (Fig. 2d); borde posterior recto. Con 2 sedas en el borde posterior de cada placa genital.

Metasoma. Proporción de la longitud del metasoma con respecto a la longitud total del cuerpo de 0.65 , evidentemente mayor que en las hembras (Cuadro 2), diferencia debida al dimorfismo sexual que exhibe el género. No hay otras diferencias evidentes. Ventralmente hay 10 sedas grandes en todos los segmentos, con un patrón regular consistente en un par medio basal y uno medio distal, formados por 1 seda a cada lado de la quilla ventral submediana, seguidos hacia la base por un par de sedas localizadas 1 a cada lado del extremo proximal; distalmente un par de sedas localizado lateralmente por encima de las quillas ventrolaterales y un par más en posición media sobre el borde distal de cada segmento (Fig. 2e). De manera más general se puede resumir en 6 sedas en la mitad distal y 4 en la basal de cada segmento.

Vesícula y aguijón. Vesícula alargada con tubérculo subaculear cónico y bien desarrollado, con la punta dirigida hacia la parte media del aguijón (Fig. 2f), lo que le diferencia de C. tecomanus (Fig. 2g).

Pedipalpos. Patrón tricobotrial A-alfa, típico del género.. Delgados, esbeltos con manos delicadas, 1.8 veces más largas que anchas y casi tan altas como anchas (Cuadro 2). Ambos dedos son largos, 1.5 veces la longitud de la mano, con 3-4 dientes accesorios basales pequeños y el resto alternando dientes grandes con 2 o 3 más pequeños. Con 8 hileras imbricadas de dientes internos (incluyendo la pequeña distal de 6 dientecillos). Ventralmente, las coxas de los pedipalpos con 8 sedas grandes, 7 en el borde anterior y una más en el posterior y con 21-22 sedas 
Cuadro 2. Medidas tomadas al holotipo y paratipo hembra (CNAN T0391) de Centruroides hirsutipalpus sp. nov., provenientes de la localidad tipo (Minatitlán, Colima: 19²2.087’ N; -104 05.128’ O)

\begin{tabular}{|c|c|c|c|}
\hline \multirow[t]{2}{*}{ Holotipo macho } & \multicolumn{3}{|c|}{ (longitud corporal $=63.04 \mathrm{~cm})$} \\
\hline & longitud (mm) & ancho $(\mathrm{mm})$ & alto $(\mathrm{mm})$ \\
\hline Cefalotórax & 5.64 & 5.11 & \\
\hline Mesosoma incluyendo SMVII & 17.20 & & \\
\hline Segmento Mesosomal VII & 4.85 & & \\
\hline Conteo pectinal & $22-22$ & & \\
\hline Segmento Caudal I & 5.44 & 2.77 & \\
\hline Segmento Caudal II & 6.55 & 2.69 & \\
\hline Segmento Caudal III & 7.24 & 2.72 & \\
\hline Segmento Caudal IV & 7.72 & 2.72 & \\
\hline Segmento Caudal V & 8.26 & 2.67 & \\
\hline Vesícula & 3.35 & 2.31 & 2.06 \\
\hline Aguijón & 1.88 & & \\
\hline Metasoma incluyendo vesícula & 38.56 & & \\
\hline Fémur & 5.74 & 1.47 & \\
\hline Patela & 6.15 & 1.91 & \\
\hline Mano & 4.01 & 2.39 & 2.24 \\
\hline Dedo fijo & 5.46 & & \\
\hline Dedo Móvil & 6.10 & & \\
\hline Base de los Peines & 0.84 & 1.32 & \\
\hline Proporción L/A de la base de los peines & 0.64 & & \\
\hline Proporción LCorporal/Lmetasoma & 1.59 & & \\
\hline Paratipo hembra & longitud corp & $55.19 \mathrm{~cm}$ & \\
\hline Cefalotórax & 5.71 & 5.61 & \\
\hline Mesosoma incluyendo SMVII & 19.61 & & \\
\hline Segmento Mesosomal VII & 5.18 & & \\
\hline Conteo pectinal & $19-20$ & & \\
\hline Segmento Caudal I & 4.22 & 3.05 & \\
\hline Segmento Caudal II & 5.05 & 2.92 & \\
\hline Segmento Caudal III & 5.38 & 2.87 & \\
\hline Segmento Caudal IV & 5.92 & 2.74 & \\
\hline Segmento Caudal V & 6.65 & 2.67 & \\
\hline Vesícula & 2.64 & 2.21 & 2.06 \\
\hline Aguijón & 2.18 & & \\
\hline Metasoma incluyendo vesícula & 29.87 & & \\
\hline Fémur & 5.44 & 1.52 & \\
\hline Patela & 6.02 & 2.13 & \\
\hline Mano & 3.61 & 2.39 & 2.06 \\
\hline Dedo fijo & 5.64 & & \\
\hline Dedo Móvil & 6.17 & & \\
\hline Base de los Peines & 0.71 & 1.40 & \\
\hline Proporción L/A de la base de los peines & 0.51 & & \\
\hline Proporción Lcorporal/Lmetasoma & 1.85 & & \\
\hline
\end{tabular}




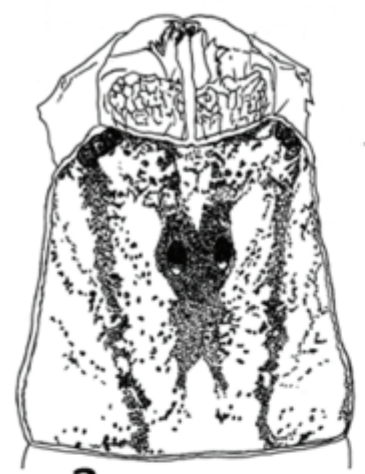

a

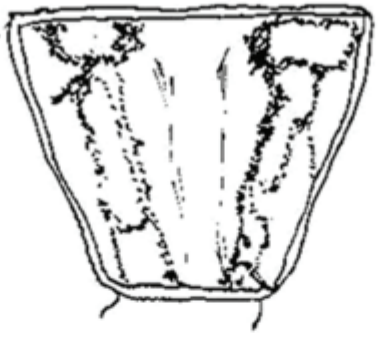

b

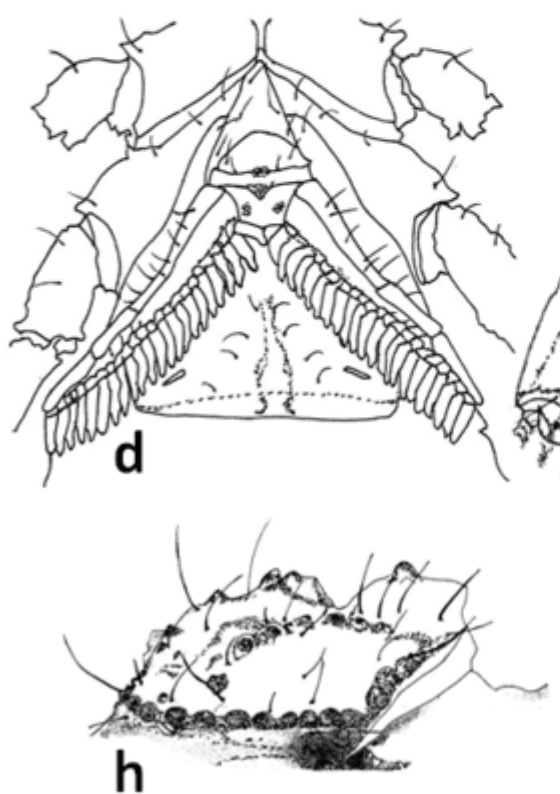

h

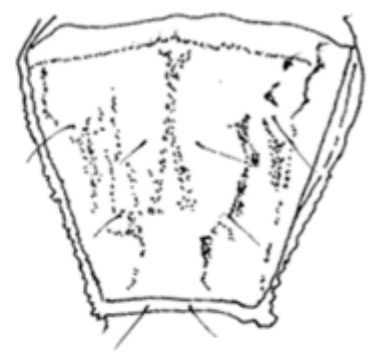

C

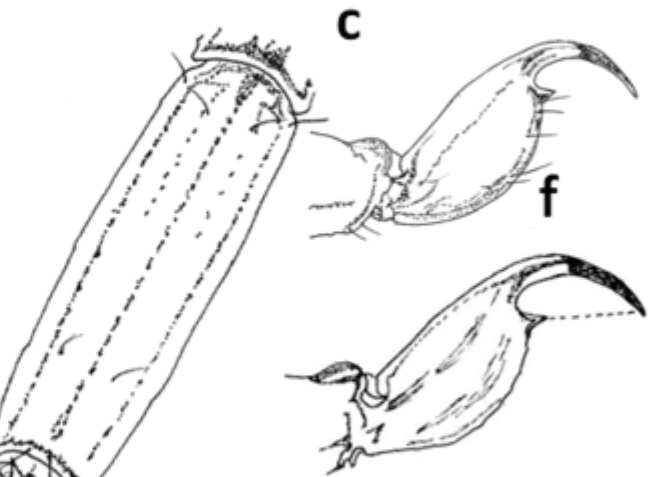

g

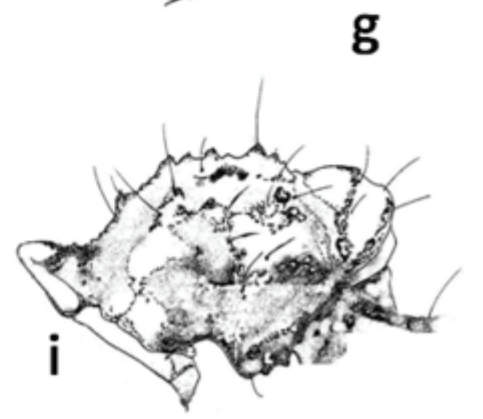

Figura 2. Características morfológicas de C. hirsutipalpus sp. nov. a, caparazón cefalotorácico; b, vista dorsal del segmento mesosomal VII; c, vista ventral del segmento mesosomal VII; d, vista ventral del mesosoma mostrando la estructura pectinal; e, vista ventral del segmento caudal V; f, vesícula y aguijón; g, vesícula y aguijón de C. tecomanus mostrando la orientación del tubérculo subaculear; $h$, vista ventral del trocánter del pedipalpo; i, vista dorsal del trocánter del pedipalpo.

sedas delicadas, en su mayoría ubicadas a los lados de la quilla digital (Fig. 3d). Mano con sedas pequeñas distribuidas irregularmente en toda la superficie. Dedos con hileras de sedas que los recorren en toda su longitud, formadas por 7-8 sedas espaciadas sobre los dientes accesorios, 1 por cada hilera justo antes del dientecillo más grande.

Medidas del holotipo macho $y$ paratipo hembra (véase Cuadro 2)

Variación. Se puede encontrar diferencia aparente entre las hembras y los machos en la sobre la cara interna de los trocánteres (Figs. $2 \mathrm{~g}$ y $2 \mathrm{~h}$ ), contrastando con las 2 o 3 que se aprecian en las coxas de las patas y las 3 o 4 de los trocánteres en otras especies cercanas. Fémur en cara externa con 11 sedas, 8 en la región distal después de al tricobotria $e 2$, y 3 sedas más en la región basal. Sedas ubicadas cerca de los bordes de las quillas, las cuales son granuladas. En la cara superior se cuentan 3-4 sedas grandes sobre el borde anterior y una más en la porción distal; cara interna con 9 sedas grandes y 4 o 5 más pequeñas (Fig. 3a y b). Patela también con muchas sedas grandes. Cara interna con 13-15 sedas, 9 de ellas grandes y las demás pequeñas y débiles, la mayoría ubicadas en la mitad proximal. Cara superior con 5 sedas grandes fácilmente visibles y 7 de menor tamaño ubicadas sobre el borde posterior. Se aprecian también 3 tricobotrias posteriores a la quilla media, 1 basal, 1 media y 1 distal, además de una cuarta tricobotria en posición distal y anterior a la quilla media (Fig. 3 c). Cara externa con 14 forma de la base de los peines, que en los machos presenta una fuerte escotadura media y es claramente más ancha en su margen anterior que en el posterior, y el margen posterior presenta además un lóbulo medio, mientras que en las hembras, el margen anterior es casi recto, al igual que el posterior, dando una forma claramente rectangular (Figs. 3f y 3g). Morfométricamente, esta diferencia se manifiesta en la proporción entre la longitud y el ancho de la base de los peines que en hembras tiene valores cercanos a 0.5 y alrededor de 0.6 en los machos (Cuadros 2 y 3). La diferencia en la proporción entre la longitud corporal y la del metasoma también es clara, con valores más bajos para los machos que reflejan el mayor tamaño del metasoma en comparación con el total del cuerpo del animal. En cuanto a las sedas, la variación que se encuentra entre hembras y machos es similar; en ambos casos en las coxas de los pedipalpos se encuentran 8-9 sedas con 6-8 sobre el borde anterior y 1-2 en el posterior; trocánteres con 17-24 sedas 

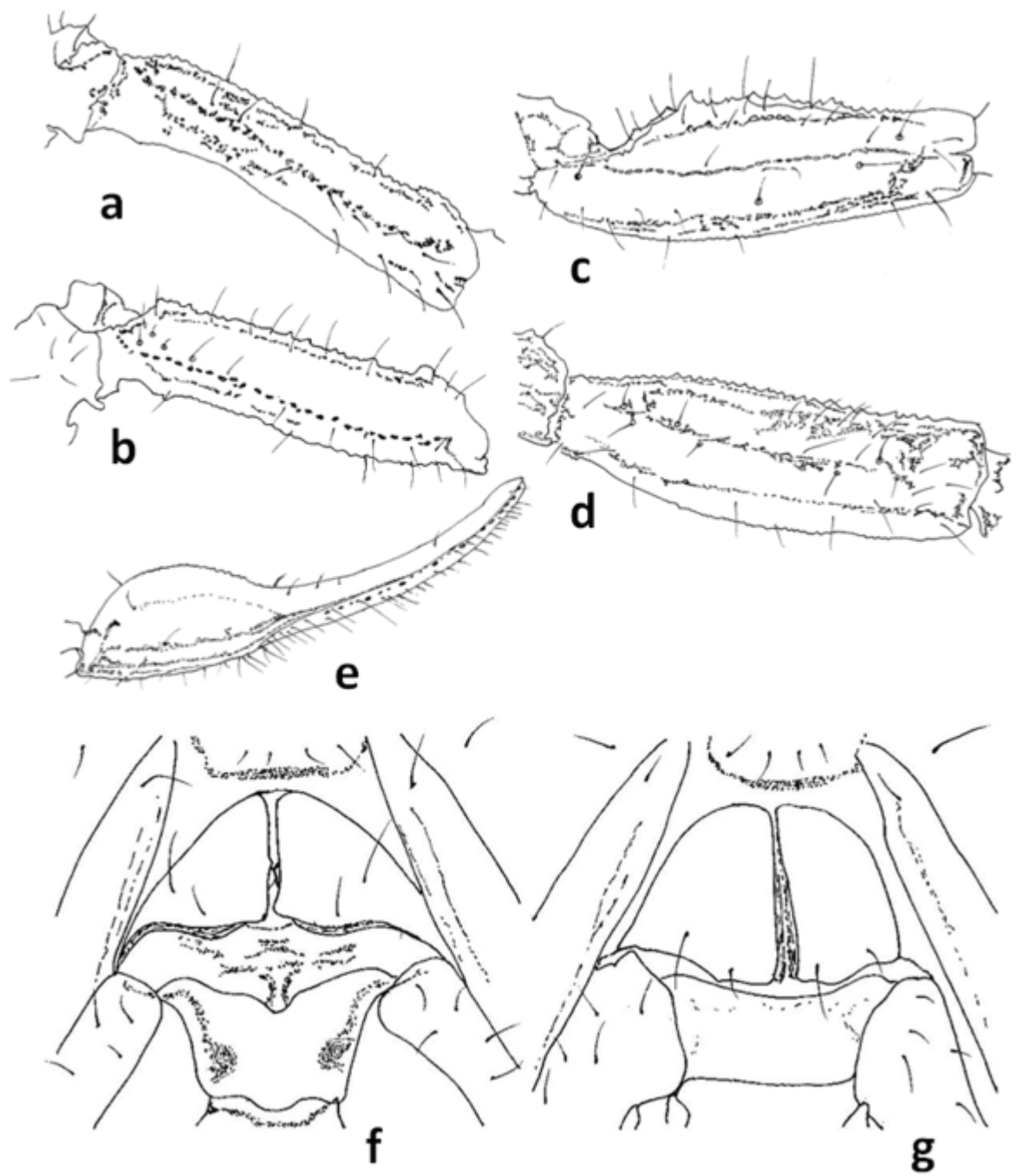

Figura 3. Características morfológicas de $C$. hirsutipalpus sp. nov. a, cara externa del fémur del pedipalpo; $b$, cara superior del fémur del pedipalpo; c, cara superior de la patela del pedipalpo; d, cara externa de la patela del pedipalpo; e, vista doral de la mano del pedipalpo; f, placas genitales y base de los peines del macho; g, placas genitales y base de los peines de la hembra.

variable con 18-24 sedas; la cara interna también tiene diferencia en la variación, en machos se observaron 11-17 sedas con 8-9 de ellas grandes y aparentes, mientras que en las hembras sólo se contaron 10-14 sedas, al menos con 7 de ellas grandes.

\section{Resumen taxonómico}

Material tipo. El holotipo es un macho adulto recolectado en Balneario El Salto, en Minatitlán, municipio del mismo nombre en el estado de Colima, México (19² 22.087' $\mathrm{N}$ y $\left.104^{\circ} 05.128^{\prime} \mathrm{O}\right)$, el 26 de mayo de 2006 por Oscar Francke, Carlos Santibáñez, Alejandro Valdéz, Héctor

(generalmente 20-21). En el fémur, la cara externa con 1117 sedas, 11-14 distales en los machos y 9-12 en el caso de las hembras y 2-4 basales en machos por 2-3 en las hembras; la cara interna con 12-16 sedas, 9-10 de ellas grandes y conspicuas en los machos mientras que en las hembras se encontraron 13-17 sedas y 11-12 de ellas grandes. La cara superior en los machos presentó menor número de sedas con 4-7, con un máximo de 5 en el borde anterior, mientras que en las hembras se contaron de 6-9 sedas de las cuales hasta 7 se localizaron en el borde anterior, pero en todos los casos sólo 1-2 se localizan en posición distal. La patela de los machos presenta en la cara superior 12-15 sedas, al menos 5 de ellas grandes, pudiendo ser hasta 10, mientras que en las hembras estas sedas pueden ser 12-14 con 810 de ellas grandes; en la cara externa en los machos se observa mayor variación con 12-23 sedas, mientras que en las hembras el número es mayor y aparentemente menos
Montaño y Jesús A. Ballesteros y será depositado en la Colección Nacional de Arácnidos del Instituto de Biología de la UNAM con el número CNAN-T0390. Un paratipo hembra también se depositará en la misma colección con el número CNAN-T0391. También se depositará un paratipo macho y uno hembra en la colección del American Museum of Natural History de Nueva York, así como en el Instituto Nacional de Referencia Epidemiológica (INDRE) y los 2 paratipos restantes pasarán a formar parte de la colección de alacranes de la Facultad de Biología de la Universidad Michoacana de San Nicolás de Hidalgo.

Distribución. Actualmente sólo se conoce de la localidad tipo.

Etimología. El nombre específico se refiere a la característica de tener un número comparativamente mayor de sedas sobre la cara dorsal de fémur y patela del pedipalpo, la cual distingue a esta especie de los demás 
Cuadro 3. Medidas de 6 paratipos de Centruroides hirsutipalpus sp. nov.

\begin{tabular}{|c|c|c|c|}
\hline \multirow{2}{*}{$\begin{array}{l}\text { Paratipos machos } \\
\text { Ejemplar } 1 \\
\text { Cefalotórax }\end{array}$} & \multicolumn{2}{|c|}{$\begin{array}{l}\text { longitud }(\mathrm{mm}) \quad \text { ancho }(\mathrm{mm}) \\
\text { longitud corporal }=61.39 \mathrm{~cm}\end{array}$} & \multirow[t]{2}{*}{ alto $(\mathrm{mm})$} \\
\hline & 5.86 & 5.58 & \\
\hline Mesosoma incluyendo SMVII & 16.20 & & \\
\hline Segmento Mesosomal VII & 4.40 & & \\
\hline Conteo pectinal & $23-23$ & & \\
\hline Segmento Caudal I & 5.66 & 2.92 & \\
\hline Segmento Caudal II & 7.00 & 2.71 & \\
\hline Segmento Caudal III & 7.62 & 2.71 & \\
\hline Segmento Caudal IV & 8.10 & 2.76 & \\
\hline Segmento Caudal V & 9.20 & 2.90 & \\
\hline Vesícula & 3.40 & 2.28 & 2.40 \\
\hline Aguijón & 1.80 & & \\
\hline Metasoma incluyendo vesícula & 40.98 & & \\
\hline Fémur & 5.89 & 1.3 & \\
\hline Patela & 6.55 & 2.13 & \\
\hline Mano & 4.16 & 2.20 & 2.40 \\
\hline Dedo fijo & 6.14 & & \\
\hline Dedo Móvil & 6.62 & & \\
\hline Base de los Peines & 0.81 & 1.20 & \\
\hline Proporción L/A de la base de los peines & 0.68 & & \\
\hline Proporción Lcorporal/Lmetasoma & 1.54 & & \\
\hline Ejemplar 2 & \multicolumn{2}{|c|}{ longitud corporal $=51.44 \mathrm{~cm}$} & \\
\hline Cefalotórax & 4.85 & 4.47 & \\
\hline Mesosoma incluyendo SMVII & 13.26 & & \\
\hline Segmento Mesosomal VII & 4.95 & & \\
\hline Conteo pectinal & $23-22$ & & \\
\hline Segmento Caudal I & 4.70 & 2.54 & \\
\hline Segmento Caudal II & 5.64 & 2.49 & \\
\hline Segmento Caudal III & 6.02 & 2.46 & \\
\hline Segmento Caudal IV & 6.63 & 2.46 & \\
\hline Segmento Caudal V & 7.37 & 2.49 & \\
\hline Vesícula & 2.97 & 2.03 & 1.83 \\
\hline Aguijón & 1.63 & & \\
\hline Metasoma incluyendo vesícula & 33.32 & & \\
\hline Fémur & 5.08 & 1.27 & \\
\hline Patela & 5.69 & 1.75 & \\
\hline Mano & 3.61 & 2.13 & 2.03 \\
\hline Dedo fijo & 4.70 & & \\
\hline Dedo Móvil & 5.33 & & \\
\hline Base de los Peines & 0.51 & 1.14 & \\
\hline Proporción L/A de la base de los peines & 0.44 & & \\
\hline Proporción Lcorporal/Lmetasoma & 1.54 & & \\
\hline Ejemplar 3 & longitud cort & $52.20 \mathrm{~cm}$ & \\
\hline Cefalotórax & 4.86 & 4.75 & \\
\hline Mesosoma incluyendo SMVII & 15.39 & & \\
\hline
\end{tabular}


Segmento Mesosomal VII

4.22

21-22

Conteo pectinal

4.45

5.21

Segmento Caudal II

6.01

Segmento Caudal III

Segmento Caudal IV

6.35

7.04

2.39

Segmento Caudal V

2.90

2.34

Vesícula

1.65

Aguijón

31.94

Metasoma incluyendo vesícula

4.94

5.56

3.91

4.72

5.66

0.66

0.57

1.63

Proporción L/A de la base de los
Proporción Lcorporal/Lmetasoma

Ejemplar 4

Cefalotórax

Mesosoma incluyendo SMVII

Segmento Mesosomal VII

Conteo pectinal

Segmento Caudal I

Segmento Caudal II

Segmento Caudal III

Segmento Caudal IV

Segmento Caudal V

Vesícula

Aguijón

Metasoma incluyendo vesícula

Fémur

Patela

Mano

Dedo fijo

Dedo Móvil

Base de los Peines

Proporción L/A de la base de los peines

Proporción Lcorporal/Lmetasoma

\section{Ejemplar 1}

Cefalotórax

Mesosoma incluyendo SMVII

Segmento Mesosomal VII

Conteo pectinal

Segmento Caudal I

Segmento Caudal II

Segmento Caudal III

0.51

1.61

longitud corporal $=51.08 \mathrm{~cm}$ 5.87

15.60

3.68

21-21

4.27 longitud corporal $=60.02 \mathrm{~cm}$

5.51

17.30

5.05

22-23

5.33

6.17

2.64

6.91

7.44

8.00

3.35 


\begin{tabular}{|c|c|c|c|}
\hline Segmento Caudal IV & 5.89 & 2.74 & \\
\hline Segmento Caudal V & 6.53 & 2.64 & \\
\hline Vesícula & 2.49 & 2.08 & 1.91 \\
\hline Aguijón & 2.39 & & \\
\hline Metasoma incluyendo vesícula & 29.62 & & \\
\hline Fémur & 5.28 & 1.45 & \\
\hline Patela & 5.82 & 2.08 & \\
\hline Mano & 3.43 & 2.26 & 2.01 \\
\hline Dedo fijo & 5.44 & & \\
\hline Dedo Móvil & 6.22 & & \\
\hline Base de los Peines & 0.64 & 1.63 & \\
\hline Proporción L/A de la base de los peines & 0.39 & & \\
\hline Proporción Lcorporal/Lmetasoma & 1.72 & & \\
\hline Ejemplar 2 & \multicolumn{2}{|c|}{ longitud corporal $=54.41 \mathrm{~cm}$} & \\
\hline Cefalotórax & 5.54 & 5.41 & \\
\hline Mesosoma incluyendo SMVII & 17.45 & & \\
\hline Segmento Mesosomal VII & 4.39 & & \\
\hline Conteo pectinal & $22-23$ & & \\
\hline Segmento Caudal I & 3.84 & 2.92 & \\
\hline Segmento Caudal II & 4.75 & 2.77 & \\
\hline Segmento Caudal III & 5.11 & 2.82 & \\
\hline Segmento Caudal IV & 5.74 & 2.72 & \\
\hline Segmento Caudal V & 6.48 & 2.59 & \\
\hline Vesícula & 2.51 & 1.98 & 1.88 \\
\hline Aguijón & 2.54 & & \\
\hline Metasoma incluyendo vesícula & 28.42 & & \\
\hline Fémur & 5.13 & 1.42 & \\
\hline Patela & 5.94 & 1.98 & \\
\hline Mano & 4.22 & 2.11 & 2.39 \\
\hline Dedo fijo & 4.80 & & \\
\hline Dedo Móvil & 6.10 & & \\
\hline Base de los Peines & 0.66 & 1.40 & \\
\hline Proporción L/A de la base de los peines & 0.47 & & \\
\hline Proporción Lcorporal/Lmetasoma & 1.81 & & \\
\hline
\end{tabular}

alacranes rayados descritos hasta la fecha.

\section{Comentarios taxonómicos}

Se hizo la comparación morfológica en la que se incluyó el número de sedas en pedipalpo, entre 4 especies de Centruroides, que por su distribución ( $C$. infamatus ornatus) o por su apariencia (C. limpidus) o ambas $(C$. tecomanus, $C$. elegans), pudieran confundirse con la nueva especie. La observación de las sedas requiere del uso del microscopio estereoscópico y las diferencias observadas se presentan en la Cuadro 4. En la práctica se requiere encontrar características macroscópicas que faciliten la identificación. En el caso de C. hirsutipalpus sp. nov., se hacen consideraciones sobre algunas diferencias morfológicas y de distribución, que pudieran ser útiles para separarlo.

Centruroides infamatus ornatus se ha registrado para el estado de Colima (Beutelspacher, 2000) que al igual que $C$. infamatus infamatus se distingue fácilmente de la especie nueva por la pigmentación del caparazón cefalotorácico, el cual tiene coloración oscura de manera difusa sobre la mayor parte de la superficie y por tanto, no presenta las 4 líneas bien definidas características del grupo "limpidus-elegans" (sensu González-Santillán, 


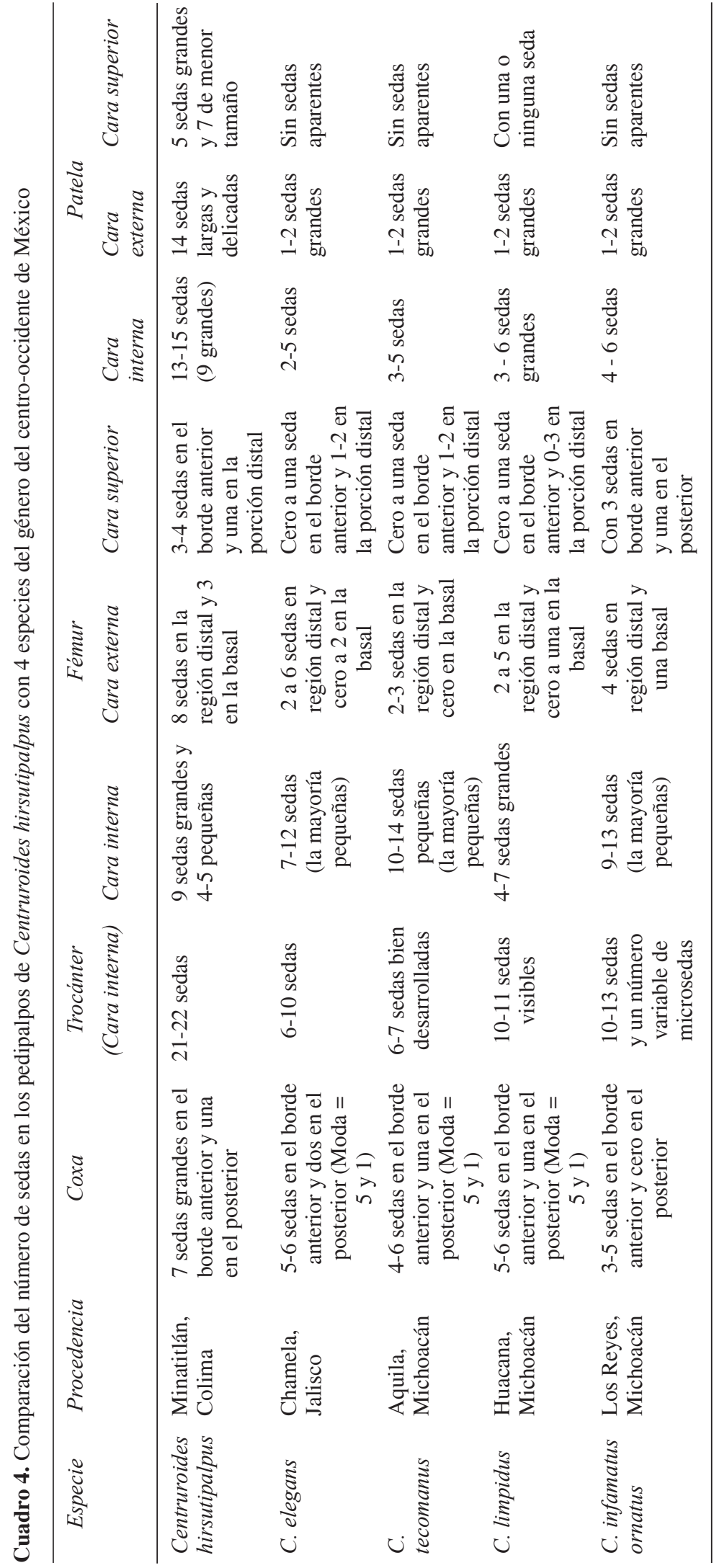

2001) y que en $C$. hirsutipalpus son evidentes (Fig. 2a). Adicionalmente se puede observar una diferencia clara en la forma de la base de los peines, los cuales en las hembras de C. hirsutipalpus presentan una muesca en el borde anterior la cual no está presente en $C$. infamatus ornatus, mientras que en el caso de los machos, el borde posterior es recto en esta especie y redondeado en el caso de la nueva especie (Fig. 4). Las otras especies comparadas presentan las 4 líneas de pigmento oscuro sobre el caparazón cefalotorácico por lo que distinguirlas es un poco más difícil.

Centruroides limpidus es conocida del centro y sur del país (Beutelspacher, 2000, Fet et al., 2000, Jaimes, 2007) y los registros que hay hacia el occidente, se ubican en el estado de Michoacán (Ponce y Beutelspacher, 2001, Ponce y Moreno, 2005, Ponce y Francke, 2004). El patrón de coloración es semejante al de la nueva especie, pero se pueden mencionar diferencias importantes en el tamaño y forma del tubérculo subaculear, el cual en $C$. limpidus siempre es pequeño y generalmente no es cónico llegando incluso a ser granular; mientras que en $C$. hirsutipalpus el tubérculo es aparente, cónico y bien desarrollado (Fig. 2f). La forma de la base de los peines también representa un criterio para diferenciar estas especies ya que en el caso de las hembras, al igual que en el caso precedente, la muesca media en el borde anterior de la base de los peines en $C$. hirsutipalpus no está presente en $C$. limpidus; mientras que en los machos, el borde posterior redondeado en la nueva especie es distinto al casi recto y ligeramente lobulado de C. limpidus (Fig. 4).

Centruroides tecomanus que se conoce del estado de Colima (Beutelspacher, 2000, Fet et al., 2000) y la zona costera del estado de Michoacán (Ponce y Beutelspacher, 2001, Ponce y Moreno, 2005, Ponce y Francke, 2004), por su apariencia general pudiera confundirse con la especie que se describe en este trabajo; sin embargo, es relativamente fácil separarlas si se considera que el tubérculo subaculear de $C$. tecomanus es cónico, está muy bien desarrollado y la punta se dirige hacia el extremo del aguijón (Ponce y Francke, 2004, Ponce et al. 2009; Fig. 2g) a diferencia del tubérculo de $C$. hirsutipalpus en el que la punta del tubérculo se orienta 


\section{MACHOS}

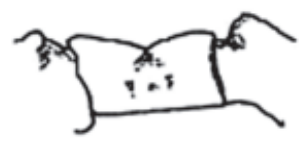

C. infamatus

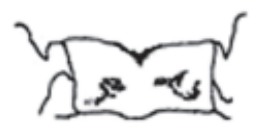

C. limpidus

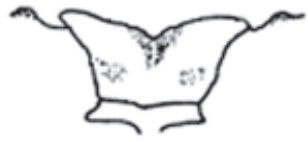

C. hirsutipalpus sp. nov.

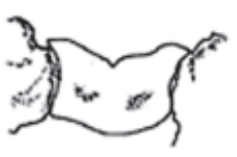

C. tecomanus

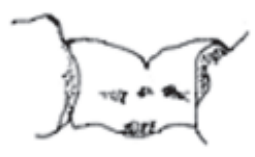

C. elegans
HEMBRAS

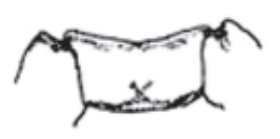

C. infamatus

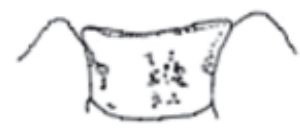

C. limpidus

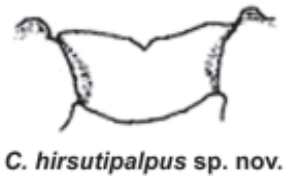

C. hirsutipalpus sp. nov.

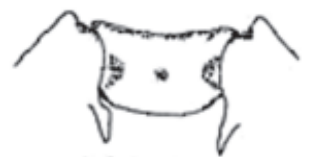

C. tecomanus

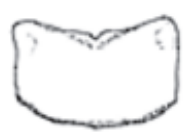

C. elegans
Figura 4. Comparación de la forma de la base de los peines de 4 especies con las que pudiera confundirse a $C$. hirsutipalpus sp. nov.

manchas. En este caso, la base de los peines de $C$. elegans sólo es ligeramente distinta de las hembras de $C$. hirsutipalpus en que el borde anterior presenta una muesca media comparativamente más tenue (Fig. 4). La relevancia de poder distinguir a las especies aquí comparadas radica en que todas ellas son de importancia médica.

Importancia médica. Se carece de estudios toxicológicos de la nueva especie. Sin embargo, datos proporcionados por el Centro Nacional de Vigilancia Epidemiológica indican que en el Municipio de Minatitlán hubo 2251 casos de picadura por alacrán en los 10 años de 1998 a 2007, y que no se registraron decesos durante ese periodo. Los datos del censo de la población (INEGI) para 2005 indican 7478 habitantes para dicho municipio, lo cual arroja un índice de morbilidad anual de aproximadamente el $3 \%$ (225 picaduras en promedio cada año, dividas entre los 7478 habitantes).

\section{Agradecimientos}

A Carlos Santibáñez, Alejandro Valdez, Héctor Montaño y Jesús A. Ballesteros, por el apoyo en la recolección

hacia la parte media del aguijón (Fig. 2). La forma de la base de los peines también ayuda en la separación. si se observa que en C. tecomanus, la base de los peines en los machos es muy similar, pero en las hembras también se aprecia la diferencia observada en el borde anterior en las 2 especies antes referidas (Fig. 4).

Centruroides elegans se registra para el estado de Jalisco (Beutelspacher, 2000, Fet et al., 2000) y más recientemente en localidades del estado de Colima (Jaimes, 2007, Francke y Sissom, inédito). Esta especie se distingue de la especie nueva por la pigmentación que forman las bandas longitudinales del mesosoma, que en esta especie están formadas por una mancha oscura en el preterguito de cada una de las placas mesosomales; mientras que en C. hirsutipalpus las presenta formadas por una mancha en el preterguito y otra en el posterguito de cada placa mesosomal y con pigmentación difusa entre ambas de los ejemplares que respaldan esta descripción y a Ana F. Quijano Ravell por su apoyo para la elaboración de las tablas de medidas de los ejemplares. Las colectas se realizaron bajo el amparo de la Licencia de Colector Científico FAUT-0175 expedida al segundo autor por la Dirección General de Vida Silvestre de la SEMARNAT.

\section{Literatura citada}

Beutelspacher, B. C. R., 2000. Catálogo de los alacranes de México. Universidad Michoacana de San Nicolás de Hidalgo, Morelia, Michoacán, México. 175 p.

Brown, C. A. 2001. Allometry of offspring size and number in scorpions. In Scorpions 2001. In memoriam Gary A. Polis, V. Fet y P. A. Selden (eds.). British Arachnological Society, Burnham Beeches, Somerset . p. 307-315.

Brown, C. A., J. M. Davis, D. J. O'Connell y D. R. Formanowitz, 
Jr. 2002. Surface density and nocturnal activity in a west Texas assemblage of scorpions. Southwestern Naturalist 47:409-419.

Contreras-Garduño J., A. V. Peretti y A. Córdoba-Aguilar. 2006. Evidence that mating plug is related to null female mating activity in the scorpion Vaejovis punctatus. Ethology 112:152-163.

De Armas, L. F., E. Martín-Frías y J. Estévez-Ramírez. 2003. Lista anotada de las especies mexicanas del género Centruroides Marx, 1890 (Scorpiones, Buthidae). Revista Ibérica de Aracnología, 8:93-98.

De Armas, L. F. y E. Martín-Frías.1998. Presencia del género Tityopsis en México y descripción de una especie nueva (Scorpiones: Buthidae). Anales de la Escuela Nacional de Ciencias Biológicas, Instituto Politécnico Nacional, 43:4549.

Fet, V., W. D. Sissom, G. Lowe y M. E. Braunwalder. 2000. Catalog of the scorpions of the world (1758-1998). The New York Entomological Society. Nueva York. 690 p.

Francke, O. F. 1977. Scorpions of the genus Diplocentrus from Oaxaca, Mexico. Journal of Arachnology, 4:145- 200.

González Santillán, E. s. f. Escorpiones de México. www. venenonemia.org

González-Santillán, E. 2001. Catálogo de escorpiones de la Colección Nacional de Arácnidos (CNAN). Tesis, Facultad de Ciencias, Universidad Nacional Autónoma de México, México, D.F. 148 p.

Hoffmann, C. C. 1931. Monografías para la entomología médica de México. Monografía No. 2, Los escorpiones de México (primera parte): Diplocentridae, Chactidae, Vejovidae. Anales del Instituto de Biología, Universidad Nacional Autónoma de México, Serie Zoología 2:291-408.

Hoffmann, C. C. 1932. Monografías para la entomología médica de México. Monografía No. 2, Los escorpiones de México (segunda parte): Buthidae. Anales del Instituto de Biología, Universidad Nacional Autónoma de México, Serie Zoología 3:243-361.

Jaimes, A. 2007. Distribución real y potencial de los escorpiones de importancia médica de México. Tesis, Facultad de Ciencias Biológicas, Universidad Autónoma del Estado de Morelos. Cuernavaca, Morelos. 54 p.

Polis, G. A. y W. D. Sissom. 1990. Life history. In The biology of scorpions, G. A. Polis (ed.). Stanford University Press, California. p. 161-223.

Polis, G. A. 1990. Ecology. In The biology of scorpions, G. A. Polis (ed.). Stanford University Press, California. p. 247293.
Ponce, S. J., R. J. Moreno B. y E. P. Miranda L. 2003. Cortejo y comportamiento de apareamiento de Centruroides sp. nov. (Scorpiones: Buthidae) en condiciones de laboratorio. Biológicas 5:64-70.

Ponce, S. J. y C. R. Beutelspacher, B. 2001. Alacranes de Michoacán. Universidad Michoacana de San Nicolás de Hidalgo, Morelia, Michoacán. 103 p.

Ponce, S. J. y O. F. Francke. 2004. Una nueva especie de alacrán del género Centruroides Marx (1890) (Scorpiones: Buthidae) de la depresión del Balsas, México. Acta Zoológica Mexicana (n.s.) 20:221-232.

Ponce, S. J., O. F. Francke, H. Cano-Camacho y E. HernandézCalderón. 2009. Evidencias morfológicas y moleculares que validan como especie a Centruroides tecomanus (Scorpiones, Buthidae). Revista Mexicana de Biodiversidad 80:71-84.

Ponce, S. J. y R. Moreno-Barajas. 2005. El género Centruroides Marx 1890 (Scorpiones: Buthidae) en México. Biológicas 7:42-51.

Ponce, S. J. 2003. Ecología y distribución del género Centruroides Marx 1890 (Scorpiones: Buthidae), en la depresión del Balsas en el estado de Michoacán. Tesis doctorado. Universidad Autónoma de Querétaro, Querétaro. 276 p.

Rein, J. O. 2008, http://www.ub.ntnu.no/scorpion-files/ scorpionidae.php; acceso: 15.11.2008.

Santibáñez-López, C. E. y J. Ponce S. 2009. A new species of Centruroides (Scorpiones: Buthidae) from the northern mountain range of Oaxaca, Mexico. Revista Mexicana de Biodiversidad 80:321-331.

Stahnke, H. L. 1970. Scorpion nomenclature and mensuration. Entomological News 81:297-316

Vachon, M. 1974. Etude des caractères utilisés pour classer les familles et les genres de Scorpions (Arachnides). 1. La trichobothriotaxie en Arachnologie, sigles trichobothriaux et types de trichobothriotaxie chez les Scorpions. Bulletin du Muséum National d'Histoire Naturelle 3:857-958

Vachon, M. 1975. Sur l'utilisation de la trichobothriotaxie du bras des pédipalpes des scorpions (Arachnides) dans le classement des genres de la famille des Buthidae Simon. Comptes rendus hebdomadaires des séances de l' Académie des Sciences, série D 281:1597-1599

Vidal-Acosta, V. y O. F. Francke. 2009. Redescripción de Tityopsis aliciae (Scorpiones: Buthidae). Revista Mexicana de Biodiversidad 80:333-339.

Yamashita, T. 2004. Surface activity, biomass and phenology of striped scorpion, Centruroides vittatus (Buthidae) in Arkansas. Euscorpius-Occasional Publications in Scorpiology 17:25-33. 